\title{
The Ion Pair Production by Each Ionizing Agent in the Lower Atmosphere, in Tokyo
}

\author{
By M. Kawano \\ Electrotechnical Laboratory, Tokyo, Japan \\ (Manuscript received 17 Dec. 1957)
}

\begin{abstract}
The content of radioactive substances in the atmosphere near the ground was measured in Tokyo, and the mean value is $0.9 \times 10^{-16} \mathrm{curie} / \mathrm{cm}^{3}$ in the daytime and $2.3 \times 10^{-16} \mathrm{curie} / \mathrm{cm}^{3}$ at night. Using these observed values and the coefficient of eddy diffusivity estimated from the method of atmospheric electricity described in the previous paper(1), we calculated the hourly values of the content of radioactive substances. The rate of ion pair production by the $\alpha$ ray emitted by the radioactive substances in the atmosphere is estimated. The earth radiation was measured, and ion pair production by that is $(3.0 \pm 0.1) \mathrm{J}$. Using these data, the rate of total ion pair production in the atmosphere are estimated.
\end{abstract}

\section{Introduction}

The presence in the atmosphere of minute quantities of natural radioactive substances become known soon after the discovery of the phenomenon of natural radioactivity. It has been shown that this substance in the atmosphere owes its origin to uranium (U) and thorium (Th) minerals distributed in the earth's crust. The radon $(\mathrm{Rn})$ and thoron (Tn) gases formed in such diffused into the atmosphere where they decay. The decay products soon attach themselves to fine particles in the air. Hence, an aerosol collection which includes the very fine particles found in the atmosphere, possesses a small but measurable amount of natural radioactivity. The methods for the collection of radioactive substance in the atmosphere have been devised by the several authors. ${ }^{(2)(3)(4)}$ Although the observations have been carried out at many stations in the world, the observations have been carried a little ${ }^{(5)}$ in our country. The atmospheric ionization has been investigated by many authors, and the chief ionizing agents in the lower atmosphere are the radiation from the radioactive substances in the atmosphere, the penetrating radiation from the radioactive substances in the earth's crust and the cosmic ray. The strongest source of the ionization in the lower atmos- phere is radon and its decay products- $\mathrm{Ra} A$, $\mathrm{Ra} B$ and $\mathrm{RaC}$. The radon content of the atmosphere has been measured by various investigators $^{(6)(7)}$, and their mean value lies between 60 and $430 \times 10^{-18}$ curie $/ \mathrm{cm}^{3}$. The diurnal variation of the $\mathrm{Rn}$ content and its correlation with the meteoroloical condition have been observed at several place in the world ${ }^{(3)(8)(9)}$, and the diurnal variation curves of $\mathrm{Rn}$ content are similar with each other by local time. Since this gas continuously decay with a half decay period of 3.82 days, there must be a source which continuously replenish it. As to this source we may reasonably expect to be in the earth. The soil has $\mathrm{Ra}$ content with an order of magnitude of $10^{-12} \mathrm{gr}$. of $\mathrm{Ra}$ per gram of soil. Some of $\mathrm{Rn}$ produced in the soil diffuses through the interstices, or soil-capilaries into the atmosphere. A theory was proposed by Hess and Schmidt ${ }^{(10)}$ which tried to define the rate of exhalation of $\mathrm{Rn}$ from the earth necessary to maintain $\mathrm{Rn}$ in the atmosphere. This theory revised first by Schmidt $^{(11)}$ and later by Priebsch. ${ }^{(12)}$ The average rate of exhalation according to this last revision was $20 \times 10^{-18}$ curie $/ \mathrm{cm}^{2}$. sec.. And, Joly and Smith(13), Smith $^{(14)}$, and Cullen ${ }^{(15)}$ carried out the observation of the $\mathrm{Rn}$ exhalation, and found the exhalation of $20-70 \times 10^{-18}$ curie $/ \mathrm{cm}^{2}$. sec.. The results of the observations carried out 
by Hess and Vancour(16) have clarified the constitution of the ionization near the ground, and the greater part of the rate of ion pair production by radioactive substances due mainly to the $\alpha$ ray. The penetrating radiation has been investigated by a few authors (17), and the results show that the greater part of this radiation is $\gamma$ radiation, and the radiation quantity of this radiation is decided by the geological condition and the state of the ground surface.

These observations have been carried out very scarcely in our country except the special case in the field of the geochemistry.

In case of discussing the ionization equilibrium process in the lower atmosphere, the rate of ion pair production is the most important quantity. As the values of the atmospheric electric elements have local character, we must measure the rate of ion pair production at the same place with the observations of the atmospheric electric elements. But, the lobservations of these elements under the consideration mentioned above have scarcely been carried out at any place.

In this chapter, the $\mathrm{Rn}$ exhalation obtained theoretically from the observing results of the $\mathrm{Rn}$ content will be shown. And, the diurnal variation curves of the rate of ion pair production by each ionizing agent obtained by the direct and indirect method are discussed.

\section{The measuring method of radioactivity in the atmosphere}

The instrument used for the measurement of radioactive substance in the atmosphere is graphically shown in Fig. 1.

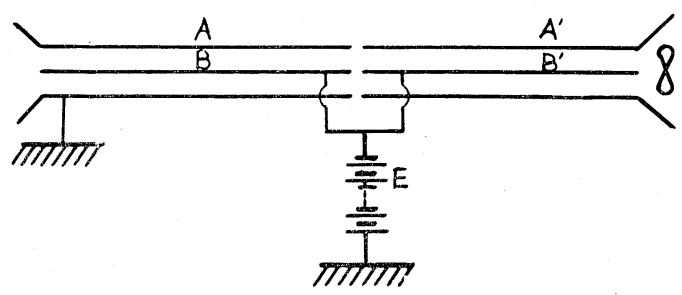

Fig. 1. The apparatus for measuring of the content of radioactive substance in the atmosphere.

The air is drawn through the outer cylinder by a motor-driven fan, $\mathrm{F}$.

The central wire, $B$, which is insulated from the air flow tube by an insulator of ebonite, is maintained at a constant potential of $V$ by battery $\mathrm{E}$. In order to determine the perfect capture of the decay products of $\mathrm{Rn}$ in the air flow by the central wire, B, a set of concentric cylinder is attached to the air flow tube, A. This set of concentric cylinder is almost the same in dimensions as the former tube, A. An electric field between an insulated central wire which is

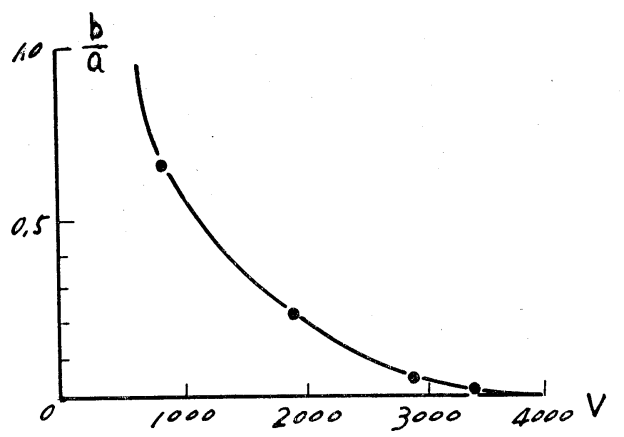

Fig. 2. The ratio of the radioactivity captured by the second central wire to that captured by the front central wire.

maintained at the same potential with the central wire of the former tube and the outer cylinder causes radioactive dusts (radioactive decay products) to be collected on the wire. If the radioactive decay products in the air flow are captured perfectly by the central wire of the front condenser, the radioactivity captured by the central wire of the second condenser may be zero. A Lauritzen electroscope is used for measuring the radioactivity captured by each central wire, and Al-foil of $30 \mu$ thickness is used for the shielding plate of electroscope. According to the measurements, the radioactivity of the radioactive dusts captured by the central wire maintained at a positive high potential is almost zero, and that captured by a negatively charged wire is measureable. So, each central wire is kept at negative high potential through this experiment.

The ratio of the radioactivity captured by the second central wire (b) to that captured by the front central wire (a) is shown in Fig. 2. The figure shows that the central wire of the front cylinder captures almost all radio- 
active dusts in the airflow at the voltage of 3,500 volts.

The relation between the limiting mobility of charged particles in airflow and the voltage of central wire is given by the following formula:

$$
k_{c}=\frac{\left(R^{2}-r^{2}\right) u}{2 l V}
$$

Here, $k_{c}$ : limiting mobility,

$$
\begin{array}{cl}
R: & \text { radius of outer cylinder, } \\
r: & \text { radius of central wire, } \\
u: & \text { velocity of airflow, } \\
V: & \text { voltage of central wire, } \\
l: & \text { length of central wire. }
\end{array}
$$

In the case of our experiment, the values of $R, r$ and $l$ are $3.0 \mathrm{~cm}, 0.01 \mathrm{~cm}$ and $120 \mathrm{~cm}$ respectively. The value of the limiting mobility of radioactive dust particle, $k_{c}$, is a function of the velocity of airflow, $u$, and the voltage of the central wire, $V$.

Considering the relation of (1), the limiting mobility of radioactive dust particle seems to be $3 \times 10^{-3} \mathrm{~cm}^{2} / \mathrm{sec}$. V.

Being based on the result of this experiment, the central wire of the front cylinder was kept at voltage of $-3,500$ volts constantly through this work.

\section{The results of the measurements of the radioactivity in the atmosphere}

For the measurement of the radioactive substances in the lower atmosphere, the aspirating tube of the instrument mentioned above is set at the height of $1 \mathrm{~m}$ above the

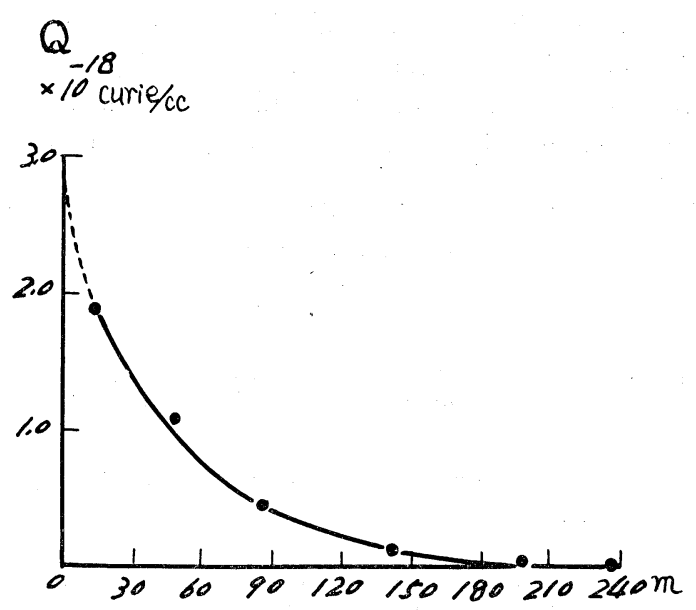

Fig. 3. Decay curve of radioactivity. ground surface. As ascertained by several authors, the radioactivity captured by the induction method is almost saturated by the aspiration of the air during about three hours. Then, the aspirating times are always three hours through these measurements, and the quantity of the aspirating air is measured by the anemometer.

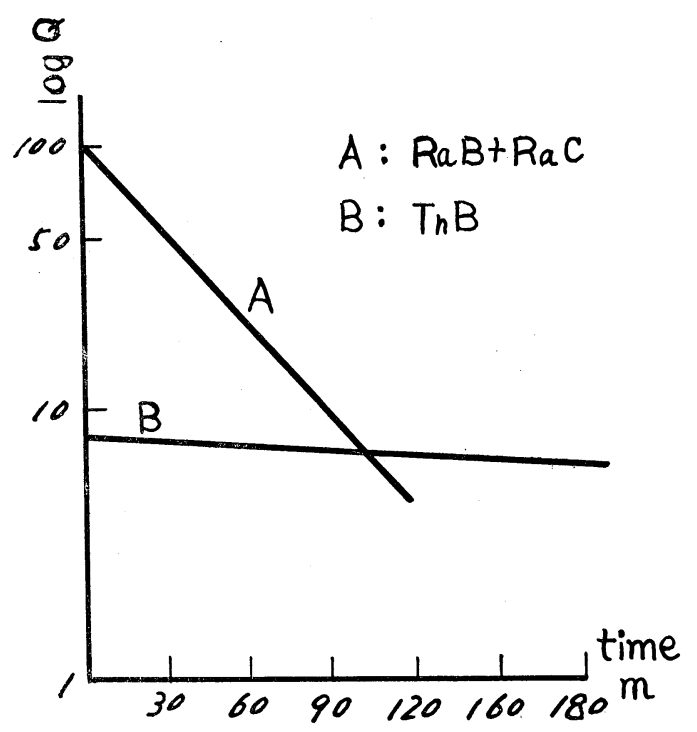

Fig. 4. The result of analysis of the decay curve.

The decay curve of radioactivity captured by the central wire is shown in Fig. 3, and the result of analysis of the curve by means of the ordinary procedure for examining the graph represented by means of a semilogarithm being shown in Fig. 4. The radioactive substances captured by the central wire are $\mathrm{RaB}, \mathrm{RaC}$ and $\mathrm{ThB}$. The quantity of $\mathrm{ThB}$ is only a few per cent of $\mathrm{Ra} B+\mathrm{RaC}$. As $\mathrm{Rn}$ gas is not collected to the central wire by the induction method, the following operation is needed for obtaining $\mathrm{Rn}$ content which is kept in the equilibrium state with its decay products. Compared with $\mathrm{Rn}$, the half decay periods of its decay products are very short. Consequently, the ratio of the number of $\mathrm{Rn}$ atoms to that of each decay product in the radioactive equilibrium state is given by the following relation $^{(17)}$ :

$$
A_{1} / A_{i}=\tau_{i} / \tau_{1} .
$$

Here, $\tau_{1}$ and $\tau_{i}$ are the decay constants of 
Rn and its decay product, and $A_{1}$ and $A_{i}$ are the number of atoms in the unit volume of the air. Using the relation of (2), we get the number of atoms of $\mathrm{Rn}$ as follows.

$$
A_{1}=\left(A_{2}+A_{3}\right) \frac{\tau_{2} \tau_{3}}{\tau_{1}\left(\tau_{2}+\tau_{3}\right)} .
$$

Here, the suffixs of 2,3 correspond to $\mathrm{Ra} B$ and $\mathrm{Ra} C$ respectively. The values of each decay constant are shown in Table 1. Table 2 shows the results of observation of the content of $\mathrm{RaB}+\mathrm{RaC}$ in the daytime and at night from the summer to the autumn of 1953 at Nishigahara, Tokyo.

The values of $\mathrm{Rn}$ content shown in the same table are calculated by the relation of (3). As shown in Table 2, the content of the radioactive substance near the ground in the daytime is different from that at night. The seasonal difference between summer and autumn is not so remarkable.

Table 1. Decay constants of Rn series

\begin{tabular}{l|l}
\hline & decay constant \\
\hline $\mathrm{Rn}$ & $2.09 \times 10^{-6} \mathrm{sec}^{-1}$ \\
$\mathrm{RaB}$ & $4.31 \times 10^{-4}$ \\
$\mathrm{RaC}$ & $5.89 \times 10^{-4}$
\end{tabular}

Table 2. The observed values of $\mathrm{RaB}+\mathrm{RaC}$, and the estimated ones of $\mathrm{Rn}$

\begin{tabular}{l|c|c}
\hline & daytime & night \\
\hline $\mathrm{RaB}+\mathrm{RaC}$ & $\begin{array}{c}0.75 \times \\
10^{-18} \mathrm{curie} / \mathrm{cm}^{3}\end{array}$ & $\begin{array}{c}1.92 \times \\
10^{-18} \mathrm{curie} / \mathrm{cm}^{3}\end{array}$ \\
\hline $\mathrm{Rn}$ & $0.9 \times 10^{-16}$ & $2.3 \times 10^{-16}$ \\
\hline
\end{tabular}

\section{The content of radioactive gas emanat- ed from the earth's surface}

If $S$ is the rate of exhalation in curie $/ \mathrm{cm}^{2}$. sec., and $Q$ the radioactive content in curie/ $\mathrm{cm}^{3}$, we can derive from the continuity condition an expression for equilibrium in a surface layer column of air of unit cross section.

$$
S=\tau \int_{h}^{\infty} Q d z
$$

Where $\tau$ is the decay constant of $\mathrm{Rn}$ (= $\left.2.09 \times 10^{-6} \mathrm{sec}^{-1}\right)$. The vertical distribution of $\mathrm{Rn}$ content in the lower atmosphere may be able to get the following equation:

$$
K \frac{d^{2} Q}{d z^{2}}=\tau Q
$$

Boundary conditions for this equation are as follows:

$$
\begin{array}{lll}
Q=Q_{h} & \text { at } & z=h \\
Q=0 & \text { at } & z=\infty .
\end{array}
$$

The solution of the equation (5) is as follows:

$$
Q=Q_{h} e^{-\sqrt{\tau / K}(z-h)} .
$$

Substituting (6) into (4), we get the following formula:

$$
\tau \int_{h}^{\infty} Q_{h} e^{-\sqrt{\tau / K}(z-h)} d z=S .
$$

Calculating this formula, we obtain the following relation:

$$
Q_{h}=\frac{S}{\sqrt{\tau K}} e^{-\sqrt{\tau / K} h} .
$$

As our observation of $\mathrm{Rn}$ content was carried out at the height of $1 \mathrm{~m}$ above the ground surface, the value of $e^{-\sqrt{\tau \mid K} h}$ may be equal to unity practically. Then, we get the following formula:

$$
Q_{h}=\frac{S}{\sqrt{\tau K}} e^{-\sqrt{\tau / K h}} \fallingdotseq \frac{S}{\sqrt{\tau K}} .
$$

The formula (9) shows that $\mathrm{Rn}$ content near the ground may inversly proportional to the square root of the coefficient of eddy diffusivity, $K$, and proportional to the mean value of $\mathrm{Rn}$ exhalation from the ground surface. This conclusion is coincident with the observed results recently obtained by Cotton(18). The value of $S$ was obtained by the following method. The mean value of $Q_{h}$ for the duration of three hours in the daytime and at night were obtained respectively

Table 3. The estimated values of the rate of exhalation of $\mathrm{Rn}$

\begin{tabular}{l|c|c|c}
\hline & $Q$ & $\cdot K^{*}$ & $S$ \\
\hline daytime & $\begin{array}{c}0.9 \times 10^{-16} \\
\text { curie } / \mathrm{cm}^{3}\end{array}$ & $\begin{array}{c}3 \times 10^{4} \\
\mathrm{~cm}^{2} / \mathrm{sec}\end{array}$ & $\begin{array}{c}21 \times 10^{-18} \\
\text { curie } / \mathrm{cm}^{2} \cdot \mathrm{sec}\end{array}$ \\
\hline night & $2.3 \times 10^{-16}$ & $5 \times 10^{3}$ & $20 \times 10^{-18}$ \\
\hline
\end{tabular}

* The values of the coefficient of eddy diffusivity, $K$, is the medial ones in the daytime and at night respectively. 
at Nishigahara (about $3 \mathrm{~km}$ apart from Hongo), and their results were shown in Table 2. We use the results obtained at Nishigahara for this investigation. The mean observed values in the daytime and at night are used here for $Q_{h}$. Using the formula (9) and the mean values of $K$ estimated by the method of the atmospheric electricity corresponding to the observation time of $Q_{h}$ in summer, we may be able to estimate the value of $S$. Table 3 shows the results in the daytime and at night respectively. According to this results, the value of $S$ estimated by the observed value of $Q_{h}$ in the daytime seems to coincide with that at night. And, this value of $S$ coincides with that obtained from the direct measurement by other investigators $^{(19)(15)}$ at different places.

\section{The diurnal variation of the content of radioactive substances and the rate of ion pair production by them}

Using the mean value of $S$ shown in Table 3 and the hourly values of $K$ shown in the previous paper(1), we have calculated the hourly values of $Q_{h}$ in each season (Fig. 5). This

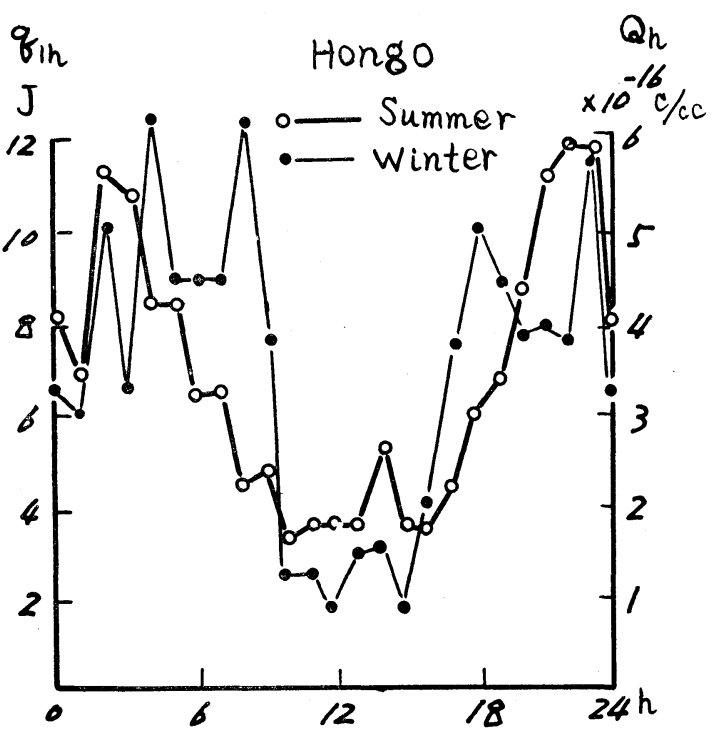

Fig. 5. The diurnal variations of the estimated content of the radioactive substances in the atmosphere and the rate of ion pair production by them.

figure shows that the maximum value occurs at $2-4 h$, and the minimum value occurs in the daytime. This characteristic of the diurnal variation curve coincides fairly well with those obtained by other investigators at different places.

In case of radioactive equilibrium, the rate of ion pair production by $\alpha$ ray radiated from $\mathrm{Rn}$ and its decay products is given by the following relation:

$$
1 \mathrm{~J}=49.8 \times 10^{-18} \text { curie } / \mathrm{cm}^{3} \text {. }
$$

Then, using the hourly values of $Q_{h}$ shown in Fig. 5, the rate of ion pair production by $\mathrm{Rn}$ and its decay products in the lower atmosphere may be obtained. Hess ${ }^{(21)}$ found that $\mathrm{Tn}$ and its decay products contribute in almost the same grade as $\mathrm{Rn}$ and its decay products to the total $\alpha$ ray ionization in the atmosphere near the ground. Considering the role of $\mathrm{Tn}$ and its decay product, the rate of ion pair production by the total radioactive substance may be estimated to be twice the value of the rate of ion pair production by $\mathrm{Rn}$ and its decay products alone.

According to this calculation, we have obtained the hourly values in each season as shown in Fig. 5.

6. The rate of ion pair production by the penetrating radiation form the ground

The total ionization produced by $\beta$ and $\gamma$ rays from the radioactive substances of the $\mathrm{U}$ and $\mathrm{Th}$ families and $\mathrm{K}$ contained in rocks and soil material is usually determined by placing an ionization chamber first over the absorbing plate and then over the ground. The difference of the ionization observed in both cases gives the "earth radiation." The

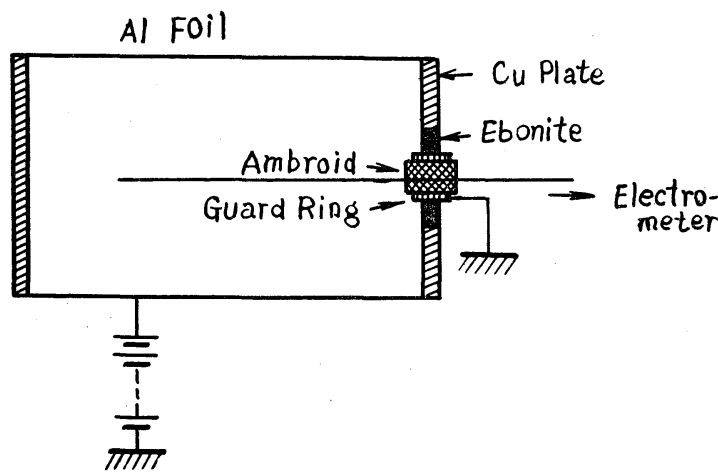

Fig. 6. The ionization chamber used for the measuring the earth radiation. 
measurement of these $\beta$ and $\gamma$ rays was carried out by the following method in the compound of Geophysical Institute, Tokyo University, Hongo.

The ionization chamber used for this measurement is shown schematically in Fig. 6. The walls of the chamber are aluminium foil of $40 \mu$ thickness. The chamber is made airtight, and the air within it, when the final sealing is done, remain there until the aluminium foil requires replacing. The walls of the ionization chamber are insulated and maintained at a constant potential. The inner electrode, $2 \mathrm{~mm}$ in diameter, is supported at the wall of the chamber by a shielded insulator. Guard ring is connected to the earth, and leak across the amber supporting the inner electrode is thus minimized. An electric field consisted between an insulated inner electrode and the wall causes ions of one sign to collect on the electrode. A high sensitive quadrant electrometer (Compton type) connected to the electrode by a fine shielded wire measures the charge accumulated, to indicate the rate of ion pair production. The sensitivity of the electrometer is $1.5 \mathrm{mV} / \mathrm{mm}$.

The characteristic curve of this chamber is shown Fig. 7. A potential difference of 800 volts between the chamber wall and inner electrode is needed for obtaining the saturation current. The electrostatic capacity of the apparatus (chamber and electrometer) is $18.0 \mathrm{~cm}$ and the volume of the chamber is 5.5 litres. For the calibration of the rate of ion pair production in this chamber, $\gamma$ ray radiated from $\mathrm{Ra}(3.9 \mathrm{mC})$ situated at $1 \mathrm{~m}$ apart from the chamber was used.

The absorption coefficients of the hard $r$ rays from $\mathrm{RaC}$ and $\mathrm{Th} \mathrm{C}^{\prime \prime}$ in lead are 0.531 and $0.472 \mathrm{~cm}^{-1}$ respectively; the $\gamma$ rays from $\mathrm{K}$ have about the same penetrating power $\left(\mu=0.516 \mathrm{~cm}^{-1}\right.$ in lead). $\gamma$ ray from $\mathrm{RaB}$ is much softer and is almost completely absorbed in $1 \mathrm{~cm}$ of lead. Other hard $\gamma$ rays are emitted by $\mathrm{UX}_{2}$, but their total intensity, compared with equilibrium amounts of other members of the $\mathrm{U}-\mathrm{Ra}$ series, is negligible. $\mathrm{Pb}$ plate of $6.0 \mathrm{~cm}$ and $6.7 \mathrm{~cm}$ thickness are needed for reduction by 5 percent of $\gamma$ ray intensity of $\mathrm{RaC}$ and $\mathrm{ThC}^{\prime \prime}$ respectively. $\mathrm{Pb}$ block of $8 \mathrm{~cm}$ thickness is used for covering $\gamma$ ray radiated from the ground. The difference of the ionization observed in both cases gives the rate of ion pair production by the $\beta$ and $\gamma$ rays from the ground. The results of measurements show that the rate of ion pair production by the penetrating radiation is $(3 \pm 0.1) \mathrm{J}$ at Hongo. The value of the penetrating radiation is constant through a day.

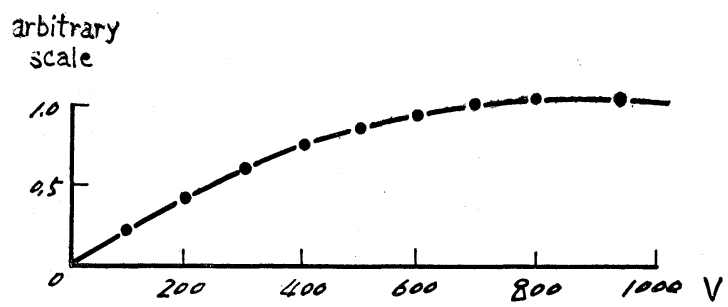

Fig. 7. The characteristic curve of the ionization chamber.

\section{The rate of ion pair production by cos- mic ray}

The rate of ion pair production by cosmic ray has been compiled by Meteorological Research Institute, ${ }^{(20)}$ and the mean value at $25^{\circ} \mathrm{N}$ is $2.35 \mathrm{~J}$, and we use this value.

Concluding the above mentioned results, we obtain the value of $5.35 \mathrm{~J}$ for the rate of ion pair production by the $\beta$ and $\gamma$ ray from the ground and cosmic ray.

Considering the hourly values of the rate of ion pair production by the radioactive substances in the atmosphere shown in Fig. 5 , we can obtain the diurnal variation of the rate of total ion pair production. This result is shown in Fig. 8.

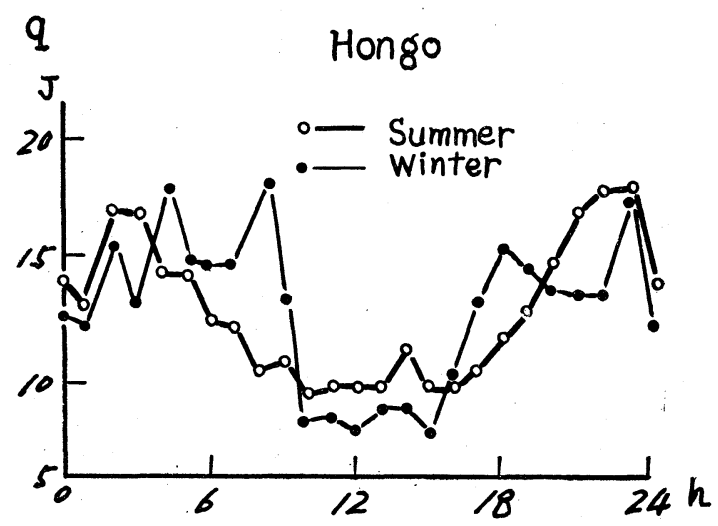

Fig. 8. The diurnal variation of the total ion pair production at Hongo in Tokyo, 


\section{Conclusion}

The content of the radioactive substances in the atmosphere was observed at Nishigahara, from the summer to the autumn of 1953. The main part of the radioactive substances captured by the induction method is $\mathrm{RaB}$ and $\mathrm{RaC}$, and this result coincides with those obtained at other stations. The limiting mobility of the radioactive carriers of $\mathrm{Ra} B, \mathrm{RaC}$ in the atmosphere is $0.003 \mathrm{~cm}^{2} / \mathrm{sec}$. V. The content of $\mathrm{Rn}$ at Nishigahara is $0.9 \times 10^{-16} \mathrm{curie} / \mathrm{cm}^{3}$ in the daytime and $2.3 \times 10^{-16} \mathrm{curie} / \mathrm{cm}^{3}$ at night. And, the seasonal difference between summer and autumn was not almost found. The rate of ion pair production by each ionizing agent were measured by the direct and indirect methods in Tokyo. According to those results, the rate of ion pair production by the $\alpha$ rays of radioactive gases and their decay products in the atmosphere is $(3-10) \mathrm{J}$, and maximum in the early morning and minimum in the daytime. And, the rate of ion pair production by the penetrating radiation from the ground is $(3 \pm 0.1) \mathrm{J}$. Considering the rate of ion pair production by cosmic ray is $2.35 \mathrm{~J}$, we can obtain the diurnal variation curve of the rate of ion pair production near the ground, and its value is $(8-16) \mathrm{J}$ at Hongo.

\section{Acknowledgement}

The author wishes to express his cordial thanks to Prof. T. Nagata, Tokyo University, for his permission of using the whole data obtained at Geophysical Institute, Tokyo University, and for his guidance and encouragement throughout this work.

\section{References}

1. Kawano, M.: In this issue.

2. Eckman, G. (1912): Jb. Radioakt., 9, 157.

3. Macek, O. and W. Illing (1953): Beitr. Geophys., 43, 388.

4. Wilkening, M. H. (1952): Nucleonics, 10, No. 6.

5. Okabe, S. (1956): Mem. College, Sci. Kyoto Univ., A, 28, 99.

6. Becker (1934): Beitr. Geophys., 42, 3 ô5.

7. Blliford, I. H. and others (1952): Rep. Naval Res. Lab., No. 4036.

8. Priebsch, J. and others (1937): Beitr. Geophys., 50, 55 .

9. Hogg, A. R. (1935): Beitr. Geophys., 43, 359.

10. Hess, V. F. and W. Schmidt (1918): Phys. Zs., 19, 109.

11. Schmidt, W. (1920): Phys. Zs., 27, 371.

12. Priebsch, J. (1931): Phys. Zs., 32, 622.

13. Joly, J. and L. B. Smith (1911): Proc. Roy. Dub. Soc., 13, 148.

14. Smith, L. B. (1912): Phil. Mag., 24, 632.

15. Cullen, T. I. (1940): Terr. Mag. Atom. Elec., $\mathbf{5 1}, 37$.

16. Hess, V. F. and R. P. Vancour (1950): Journ. Atm. Terr. Phys., 1, 13.

17. Hess, V. F. and G. A. O'Donnel (1951): Journ. Geophys. Res., 56, 557.

18. Cotton, E. (1955): Geophys. Res. Pap., No. $42,67$.

19. Zelinger, P. R. (1935): Terr. Mag. Atm. Elec., 40, 281.

20. Ishii, C. (1956): Rep. International Union. 